\title{
Exposure-response analysis of rilotumumab in gastric cancer: the role of tumour MET expression
}

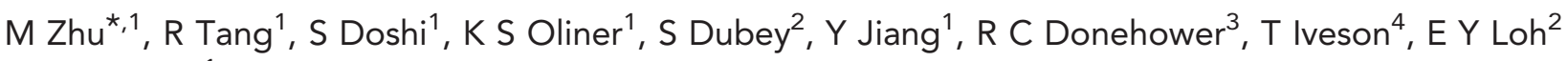 \\ and $Y$ Zhang ${ }^{1}$
}

${ }^{1}$ Translational Sciences, Amgen Inc., One Amgen Center Drive, Thousand Oaks, CA 91320, USA; ${ }^{2}$ Global Clinical Development, Amgen Inc., South San Francisco, CA, USA; ${ }^{3}$ Oncology, Johns Hopkins Medical Center, The Johns Hopkins University School of Medicine, Baltimore, MD, USA and ${ }^{4}$ Medical Oncology, University Hospital Southampton, Southampton, UK

Background: Rilotumumab, an investigational, monoclonal antibody, inhibits MET-mediated signalling. In a randomized phase 2 trial of rilotumumab \pm epirubicin/cisplatin/capecitabine in gastric or oesophagogastric junction cancer, patients receiving rilotumumab showed a trend towards improved survival, especially in MET-positive patients, but no clear dose-response relationship was observed. Exposure-response and biomarker analyses were used for dose selection and to differentiate patient subpopulations that may benefit most from treatment. Here, we analyse rilotumumab exposure-survival and exposure-safety and the impact of MET expression on these relationships.

Methods: Individual rilotumumab exposure parameters were generated using population pharmacokinetic modelling. Relationships among rilotumumab dose $\left(7.5\right.$ and $\left.15 \mathrm{mg} \mathrm{kg}^{-1}\right)$, exposure, and clinical outcomes (progression-free survival (PFS) and overall survival (OS)) were evaluated with Cox regression models and Kaplan-Meier plots. MET status and other baseline covariates were evaluated in subgroup and multivariate analyses. Treatment-emergent adverse events were summarised by exposure.

Results: Among MET-positive patients, higher rilotumumab exposure, vs placebo and low exposure, was associated with improved median PFS (80\% Cl: 7.0 (5.7-9.7) vs 4.4 (2.9-4.9) and 5.5 (4.2-6.8) months) and OS (13.4 (10.6-18.6) vs 5.7 (4.7-10.2) and 8.1 (6.9-11.1) months) without increased toxicity. No rilotumumab benefit was seen among MET-negative patients.

Conclusions: Rilotumumab had an exposure-dependent treatment effect in patients with MET-positive gastric or oesophagogastric junction cancer.

Activation of the MET receptor tyrosine kinase by its ligand, hepatocyte growth factor (HGF, also known as scatter factor), induces signalling cascades that promote cell proliferation, survival, migration, and morphogenesis (Nishiyama et al, 1994; Maulik et al, 2002; Birchmeier et al, 2003; Burgess et al, 2006). Expression of MET and/or HGF has been found in various human cancers (Taniguchi et al, 1997; Han et al, 1999; Beppu et al, 2000; Tanaka et al, 2004; Burgess et al, 2006; Drebber et al, 2008; Janjigian et al, 2011; Lennerz et al, 2011), and MET-mediated signalling pathways have been proposed as therapeutic targets in cancer (Birchmeier et al, 2003; Burgess et al, 2006; Accornero et al, 2010). In gastric cancer, higher MET expression within tumours is associated with tumour invasiveness, metastasis, and disease stage (Taniguchi et al, 1998; Nakajima et al, 1999; Amemiya et al, 2002; Drebber et al, 2008; Lennerz et al, 2011), and both MET and HGF expression within tumours were found to be negative prognostic factors (Taniguchi et al, 1998; Wu et al, 1998; Nakajima et al, 1999; Birchmeier et al, 2003; Drebber et al, 2008; Lennerz et al, 2011).

Rilotumumab is an investigational, fully human, IgG2 monoclonal antibody that binds to HGF and inhibits MET-mediated

*Correspondence: Dr M Zhu; E-mail: minz@amgen.com

Received 4 September 2014; revised 17 November 2014; accepted 8 December 2014; published online 13 January 2015

(c) 2015 Cancer Research UK. All rights reserved 0007-0920/15 
signalling pathways (Cao et al, 2001; Jun et al, 2007; Gao et al, 2009). In a double-blind, randomized phase 2 clinical trial (NCT00719550), patients received rilotumumab (7.5 or $15 \mathrm{mg} \mathrm{kg}^{-1}$ ) or placebo administered intravenously (IV) every 3 weeks in combination with epirubicin, cisplatin, and capecitabine (ECX: $50 \mathrm{mg} \mathrm{m}^{-2}$ IV day 1, $60 \mathrm{mg} \mathrm{m}^{-2}$ IV day 1 , and $625 \mathrm{mg} \mathrm{m}^{-2}$ twice a day orally on days $1-21$, respectively); rilotumumab plus ECX showed trends towards improved progression-free survival (PFS) and overall survival (OS) compared with placebo plus ECX in patients with gastric or oesophagogastric junction cancer, but no clear dose-dependent effects of rilotumumab on survival were seen (Iveson et al, 2014). Exploratory analysis suggested that high tumour MET expression was predictive of rilotumumab response.

Exposure-survival and exposure-safety analyses are commonly applied to phase 2 clinical trials to determine a therapeutic dose for confirmatory phase 3 trials in cancer research (Claret et al, 2010, 2012; Bruno et al, 2011). Combining exposure-response and biomarker analyses to identify a subset of patients who are most likely to benefit from a specific anticancer agent is still an emerging field of study. Patients in the rilotumumab phase 2 trial were not randomized according to tumour MET levels; therefore, we performed exposure-biomarker-response analyses in order to identify a subpopulation of patients who could benefit most from the treatment. The objectives of this study are to evaluate (1) the rilotumumab exposure-survival relationship; (2) any impact of tumour MET expression on the exposure-survival relationship; (3) potential confounding factors that may affect the exposuresurvival relationship; and (4) the rilotumumab exposure-safety relationship.

\section{MATERIALS AND METHODS}

Data and population pharmacokinetic model. Rilotumumab serum concentrations for the population pharmacokinetic analysis were obtained from a first-in-human, phase 1 dose-escalation study of rilotumumab in patients with advanced solid tumours (Gordon et al, 2010) and from the phase 2 double-blind study of rilotumumab plus ECX in patients with unresectable locally advanced or metastatic gastric or oesophagogastric junction adenocarcinoma (Iveson et al, 2014). All study procedures for the phase 1 and 2 studies were approved by an Institutional Review Board and done in accordance with the Declaration of Helsinki. Each patient provided written informed consent before enrolment.

General methodology for the pharmacokinetic analysis has been provided in a previous publication (Zhu et al, 2014). The population pharmacokinetic model was used to simulate individual rilotumumab exposure parameters for patients in the phase 2 study. These simulated individual rilotumumab exposure parameters and survival data (PFS, OS) obtained from the phase 2 trial were used in the exposure-response analyses.

Rilotumumab and tumour MET expression measurement. Rilotumumab serum concentrations were determined by an ELISA with a lower limit of quantitation of $31.25 \mathrm{ng} \mathrm{ml}^{-1}$ (Gordon et al, 2010). Tumour MET expression was previously identified as a potential biomarker to predict benefit from rilotumumab (Iveson et al, 2014). MET expression was determined using an immunohistochemistry assay (MET IHC pharmDx kit; Dako North America, Carpinteria, CA, USA) on archival patient tumour samples. Patients were divided into MET-positive and METnegative subgroups as described (Iveson et al, 2014). Briefly, MET positivity was defined as $\geqslant 25 \%$ membranous staining of tumour cells at any intensity, and MET negativity was defined as $<25 \%$ membranous staining.

Dose- and exposure-survival analysis. Dose-survival analysis was performed as described (Iveson et al, 2014).
Individual rilotumumab exposure parameters were generated from population pharmacokinetic analysis for exploring exposureefficacy and exposure-safety relationships. The rilotumumab exposure parameters used in these analyses were maximum serum concentration $\left(C_{\max }\right)$, minimum serum concentration $\left(C_{\min }\right)$, and area under the curve in cycle 1 and at steady state. All exposure parameters showed consistent trends in association with the survival measurements, whereas $C_{\min }$ at steady state $\left(C_{\text {minss }}\right)$ was found to have the strongest association with survival data. Therefore, $C_{\text {minss }}$ was chosen for further exposure-efficacy analyses.

Subgroup analyses were carried out based on pooled $C_{\text {minss }}$ values from patients who received 7.5 or $15 \mathrm{mg} \mathrm{kg}^{-1}$ rilotumumab, and patients were divided into low and high rilotumumab exposure groups based on median $C_{\text {minss }}$, with low exposure defined as $C_{\text {minss }}<94 \mu \mathrm{g} \mathrm{ml}^{-1}$ and high exposure defined as $C_{\text {minss }}$ $\geqslant 94 \mu \mathrm{g} \mathrm{ml}^{-1}$. Kaplan-Meier estimates were used to examine survival in different rilotumumab exposure-defined subgroups (that is, high exposure, low exposure, no exposure (placebo)). The log rank test was used to make subgroup comparison.

For the exposure-survival analysis, the placebo and low and high rilotumumab exposure groups were further subdivided into METpositive and MET-negative groups. Subgroup analyses of METpositive and MET-negative groups were conducted to evaluate the impact of tumour MET expression on the exposure-survival relationship. Cox proportional hazard models were implemented to evaluate the effect of rilotumumab exposure level on PFS and OS within the MET subgroups, and the effect of rilotumumab and MET expression on survival is illustrated by forest plots. The estimated PFS or OS hazard ratio (HR) and 95\% confidence interval (CI) for the low and high rilotumumab exposure groups $v s$ the placebo arm among patients with MET-positive and METnegative tumours were provided with the exposure level group as the independent variable and PFS/OS as the dependent variable. The interaction $P$ value for testing the heterogeneity of the MET expression effect between the exposure and placebo groups is presented.

Evaluation of potential confounding factors. To evaluate whether any potential confounding factors may affect the exposure-survival relationship, multivariate analyses with a forward selection method were used to evaluate the effect of baseline factors on PFS and OS. The covariates selected for analysis included patient baseline characteristics (region, sex, age, body weight, liver metastasis, Eastern Cooperative Oncology Group performance status, and disease extent at enrolment (locally advanced or metastatic)) and measured baseline laboratory values (total bilirubin, alanine aminotransferase (ALT), aspartate aminotransferase, alkaline phosphatase (ALP), serum creatinine, creatinine clearance $(\mathrm{CL})$, blood urea nitrogen, albumin, glucose, absolute neutrophil count (ANC), white blood cell, monocytes, phosphorus, haematocrit, haemoglobin, potassium, chloride, platelets, red blood cells, serum urea, calcium, potassium, and lymphocytes).

The effects of rilotumumab $C_{\text {minss }}$ and all the candidate baseline covariates on PFS and OS were evaluated with the placebo arm as reference. A forward proportional hazards regression analysis was used to identify terms important for predicting PFS or OS. The Wald Score $\chi^{2}$ statistics were used to assess inclusion of the terms in the model. A term was included in the model when it resulted in a score $\chi^{2}$ statistic, which satisfies the pre-specified significance level for entry criteria $=0.1$. The final model was reached when none of the remaining terms were significant at this level. The effects of $C_{\text {minss }}$ and potential prognostic factors on PFS and OS in the final model (HR, 95\% CI, $P$ value) were estimated.

Exposure-safety analysis. Patient incidence of treatment-emergent adverse events (AEs) by preferred term and worst grade was summarised by descriptive statistics in the placebo and low and high 
rilotumumab exposure groups. The relationships between changes in laboratory values of interest from baseline and rilotumumab exposure were explored using linear regression models.

Statistical considerations. These analyses were considered exploratory and hypothesis generating. $P$ values generated from the analyses were used mainly as a descriptive measure rather than to test hypotheses, and $P$ values were not corrected for multiple comparisons.

\section{RESULTS}

Patients. The phase 2 study included 121 patients; 82 patients were randomized to receive rilotumumab plus ECX, and 39 were randomized to receive placebo plus ECX. Overall, 120 patients received $\geqslant 1$ dose of rilotumumab $(n=81)$ or placebo $(n=39)$ and were included in the analyses here. Patients were divided into low and high rilotumumab exposure groups based on the median $C_{\text {minss }}\left(94 \mu \mathrm{g} \mathrm{ml}^{-1}\right)$. Of the 39 patients who received the $15 \mathrm{mg} \mathrm{kg}^{-1}$ rilotumumab dose, 7 and 32 patients were in the low- and high-exposure subgroups, respectively. Of the 42 patients who received the $7.5 \mathrm{mg} \mathrm{kg}^{-1}$ rilotumumab dose, 33 and 9 patients were in the low- and high-exposure subgroups, respectively.

Patients were $\geqslant 18$ years of age (mean $=58.8$ years), had unresectable locally advanced or metastatic gastric or oesophagogastric junction adenocarcinoma, and had not received prior systemic therapy for this disease. Baseline patient demographics and disease characteristics were generally evenly distributed among groups (Table 1).

Population pharmacokinetic analysis. A linear two-compartment model was created using data from the first-in-human study and the phase 2 study (see Materials and Methods). The model adequately described rilotumumab concentration data following IV infusion. The estimated rilotumumab population pharmacokinetic parameters are displayed in Table 2. Within the dose range from 0.5 to $20 \mathrm{mg} \mathrm{kg}^{-1}$, rilotumumab showed linear, dose-proportional, and time-independent kinetic behaviours. The estimated typical value of rilotumumab systemic CL was 0.2161 per day per $70 \mathrm{~kg}$, and the volume of distribution in the central compartment $\left(V_{1}\right)$ was 3.741 per $70 \mathrm{~kg}$. The inter-patient variability in CL was $37.5 \%$. Within the covariates examined (including baseline demographics, laboratory values, biomarkers, and disease status), body weight was

Table 1. Baseline patient and disease characteristics

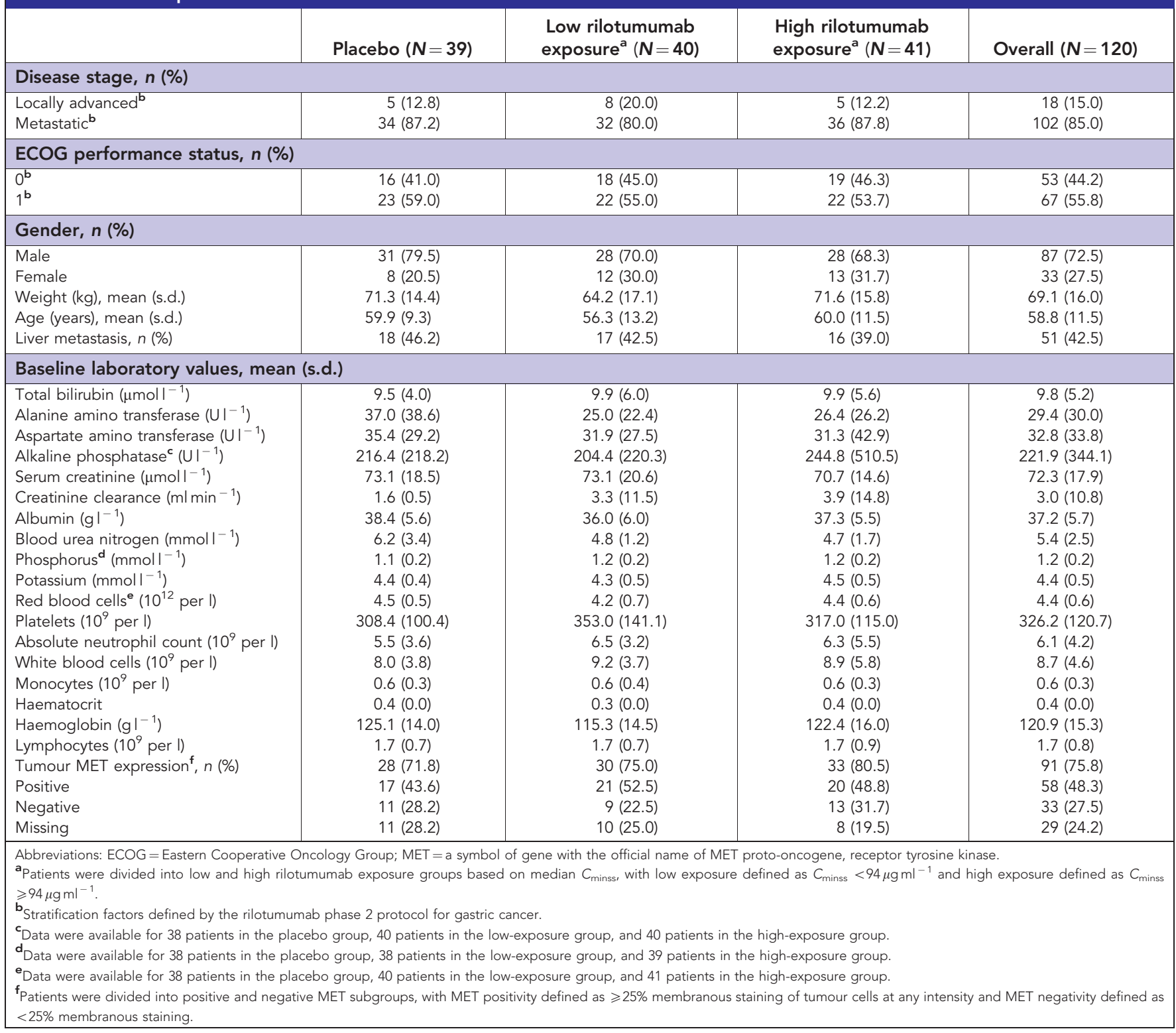


Table 2. Rilotumumab population pharmacokinetic parameter estimates

\begin{tabular}{|c|c|c|c|}
\hline Parameters & Units & Typical value (RSE) & Bootstrap mean $(95 \% \mathrm{Cl})$ \\
\hline $\mathrm{CL}$ & I per day per $70 \mathrm{~kg}$ & $0.216(4.40)$ & $0.216(0.199-0.232)$ \\
\hline WT on $\mathrm{CL}$ & $\% / 10 \mathrm{~kg}$ & $9.50(25.2)$ & $9.47(5.22-13.3)$ \\
\hline$V_{1}$ & I per $70 \mathrm{~kg}$ & $3.74(3.50)$ & $3.74(3.57-3.92)$ \\
\hline WT on V1 & $\% / 10 \mathrm{~kg}$ & $9.22(20.5)$ & $9.21(6.60-12.0)$ \\
\hline Q & I per day & $0.895(34.6)$ & $0.890(0.422-1.48)$ \\
\hline$V_{2}$ & I & $2.22(11.2)$ & $2.19(1.69-2.69)$ \\
\hline \multicolumn{4}{|c|}{ Inter-individual variability (\% CV) } \\
\hline $\begin{array}{l}\omega_{\mathrm{CL}} \\
\omega_{\mathrm{V} 1} \\
\omega_{\mathrm{Q}} \\
\omega_{\mathrm{V} 2}\end{array}$ & & $\begin{array}{r}37.5(18.5) \\
20.7(25.3) \\
105(60.2) \\
48.5(54.9)\end{array}$ & $\begin{array}{c}37.4(31.6-43.7) \\
20.7(16.1-24.5) \\
100(1.10-145) \\
51.5(29.3-73.8)\end{array}$ \\
\hline \multicolumn{4}{|l|}{ Coviariance } \\
\hline $\begin{array}{l}\rho_{\mathrm{CL}-\mathrm{V} 1} \\
\rho_{\mathrm{V} 1-\mathrm{V} 2}\end{array}$ & & $\begin{array}{l}0.0464(31.7) \\
0.0645(57.1)\end{array}$ & $\begin{array}{l}0.0468(0.0229-0.0704) \\
0.0568(0.00843-0.0948)\end{array}$ \\
\hline \multicolumn{4}{|c|}{ Residual error (\% CV) } \\
\hline $\begin{array}{l}\sigma_{\text {Intensive PK }} \\
\sigma_{\text {Sparse PK }} \\
\end{array}$ & $\begin{array}{l}24.9(1.50) \\
33.3(3.36) \\
\end{array}$ & $\begin{array}{l}24.5(19.8-28.8) \\
33.2(27.6-38.3) \\
\end{array}$ & \\
\hline
\end{tabular}

the only significant covariate on CL and $V_{1}$; both CL and $V_{1}$ increased by approximately $9-10 \%$ per each $10 \mathrm{~kg}$ increase in body weight. Primary tumour location, tumour MET expression level, plasma HGF level, and ECX co-administration did not show any significant effects on the pharmacokinetic parameters. The population pharmacokinetic model was used to simulate individual exposure levels for the exposure-survival and exposure-safety analyses.

Exposure-survival analysis. The Kaplan-Meier survival curves (PFS and OS) describing the relationships of (1) rilotumumab dose and survival; (2) rilotumumab exposure and survival; and (3) rilotumumab exposure and survival based on tumour MET expression are shown in Figure 1.

Rilotumumab dose-survival relationship. Treatment with rilotumumab 7.5 and $15 \mathrm{mg} \mathrm{kg}^{-1}$ were both associated with a trend towards improved PFS and OS compared with placebo (Figure 1A and B; Iveson et al, 2014). However, the higher dose did not exhibit longer survival than the lower dose. The median PFS ( $80 \% \mathrm{CI})$ for the placebo and 7.5 and $15 \mathrm{mg} \mathrm{kg}^{-1}$ rilotumumab arms was 4.2 (3.7-4.6), 6.8 (5.6-7.3), and 5.1 (3.9-5.7) months, respectively. The median OS (80\% CI) for these groups was 8.9 (5.7-10.6), 11.1 (9.5-12.1), and $9.7(7.8-12.5)$ months, respectively.

Rilotumumab exposure-survival relationship. Higher rilotumumab exposure was associated with a trend towards longer survival (Figure 1C and D). The median PFS (80\% CI) for the placebo and low and high rilotumumab exposure groups was 4.2 (3.7-4.6), 4.9 (4.2-6.3), and 6.9 (5.5-7.1) months, respectively. The median OS (80\% CI) for these groups was 8.9 (5.7-10.6), 9.5 (7.5-11.1), and 13.2 (10.6-14.3) months, respectively.

Rilotumumab exposure-MET-survival relationship. Tumour MET expression levels were available for 91 patients in the per protocol analysis set. Higher rilotumumab exposure was associated with a trend towards longer survival in patients with MET-positive tumours (Figure 1E and F). Among patients with MET-positive tumours, median PFS (80\% CI) for the placebo and low and high rilotumumab exposure groups was 4.4 (2.9-4.9), 5.5 (4.2-6.8), and 7.0 (5.7-9.7) months, respectively. The median OS (80\% CI) for these groups was $5.7(4.7-10.2), 8.1 \quad(6.9-11.1)$, and 13.4 (10.6-18.6) months, respectively.

No treatment benefit was seen with rilotumumab plus ECX $v s$ placebo plus ECX in patients with MET-negative tumours (Figure $1 \mathrm{G}$ and $\mathrm{H}$ ). Among patients with MET-negative tumours, median $(80 \%$ CI) PFS in the placebo and low and high rilotumumab exposure groups was $5.4(4.1-5.6), 3.5$ (1.5-7.0), and 5.3 (2.9-5.7) months, respectively. The median (80\% CI) OS in these groups was $11.5(8.5-19.5), 11.1(9.2-13.1)$, and 12.5 (6.9-14.3) months, respectively.

The effects of rilotumumab exposure on PFS or OS were assessed based on a Cox proportional hazards model within different MET expression subgroups (Figure 2). Rilotumumab had no apparent effect on survival in patients with MET-negative tumours, but rilotumumab showed an exposure-dependent treatment effect in patients with MET-positive tumours. Similar results were observed with a MET-positive subgroup expressing more MET defined as $\geqslant 50 \%$ membranous staining (data on file).

Rilotumumab dose-MET-survival relationship. The KaplanMeier survival curves (PFS and OS) describing the relationships of rilotumumab dose and survival based on tumour MET expression are shown in Figure 3. Among patients with METpositive tumours, a survival benefit was observed with rilotumumab but no clear dose-response relationship was observed. Among these patients, median PFS $(80 \% \mathrm{CI})$ for the placebo and low $\left(7.5 \mathrm{mg} \mathrm{kg}^{-1}\right)$ and high $\left(15 \mathrm{mg} \mathrm{kg}^{-1}\right)$ rilotumumab dose groups was $4.4(2.9-4.9), 6.9(5.6-8.5)$, and 5.1 (3.9-7.0) months, respectively. Median OS $(80 \% \mathrm{CI})$ for these groups was 5.7 (4.7-10.2), 11.0 (9.2-12.0), and 9.7 (7.7-13.4) months, respectively. Among patients with MET-negative tumours, no benefit of rilotumumab was observed, regardless of dose. Median $(80 \% \mathrm{CI})$ PFS in the placebo and low and high rilotumumab dose groups was 5.4 (4.1-5.6), 4.0 (3.0-7.0), and 5.3 (2.8-5.7) months, respectively. Median (80\% CI) OS in these groups was 11.5 (8.5-19.5), 12.1 (9.2-13.2), and $11.1(6.9-13.3)$ months, respectively.

Potential confounding factors of the exposure-survival analysis. In the multivariate PFS analysis, rilotumumab $C_{\text {minss }}$, serum urea, creatinine, ANC, and chloride were identified as covariates. After adjusting for the effects of urea, creatinine, ANC, and chloride, $C_{\text {minss }}$ was associated with improved PFS in the high rilotumumab 

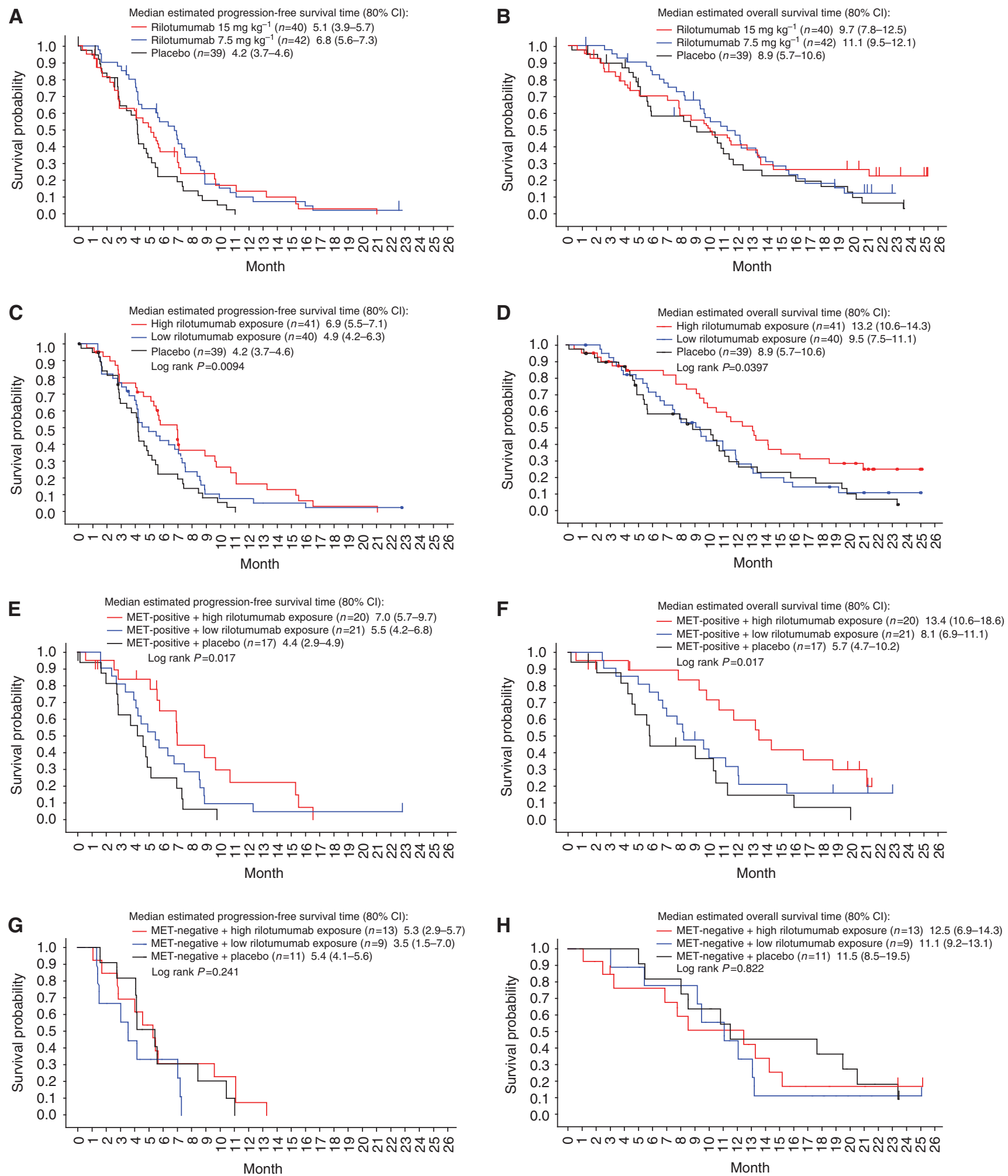

Figure 1. Kaplan-Meier analysis of progression-free survival (PFS) and overall survival (OS). PFS is shown in $\mathbf{A}, \mathbf{C}, \mathbf{E}$, and $\mathbf{G}$. OS is shown in $\mathbf{B}, \mathbf{D}, \mathbf{F}$, and $\mathbf{H}$. PFS and OS were examined based on rilotumumab dose (A, B), rilotumumab exposure (C, D), rilotumumab exposure in the MET-positive subgroup (E, F), and rilotumumab exposure in the MET-negative subgroup (G, H). Low rilotumumab exposure was defined as $C_{\text {minss }}<94 \mu \mathrm{g} \mathrm{ml}^{-1}$, and high rilotumumab exposure was defined as $C_{\text {minss }} \geqslant 94 \mu \mathrm{g} \mathrm{ml}^{-1}$. MET positivity was defined as $\geqslant 25 \%$ membranous staining of tumour cells at any intensity, and MET negativity was defined as $<25 \%$ membranous staining. $\mathrm{Cl}$, confidence interval.

exposure group compared with the placebo group $(\mathrm{HR}=0.40$; $95 \% \mathrm{CI}=0.23-0.71 ; P=0.002)$. Rilotumumab had less of an effect on PFS in the low rilotumumab exposure group compared with the placebo group $(\mathrm{HR}=0.52 ; 95 \% \mathrm{CI}=0.30-0.90$; $P=0.019)$.
In the multivariate OS analysis, rilotumumab Cminss, ALP, albumin, creatinine, age, and ANC were identified as covariates. After adjusting for the effects of ALP, albumin, creatinine, age, and ANC, higher $C_{\text {minss }}$ was associated with improved OS in the high rilotumumab exposure group compared with placebo $(\mathrm{HR}=0.32$; 


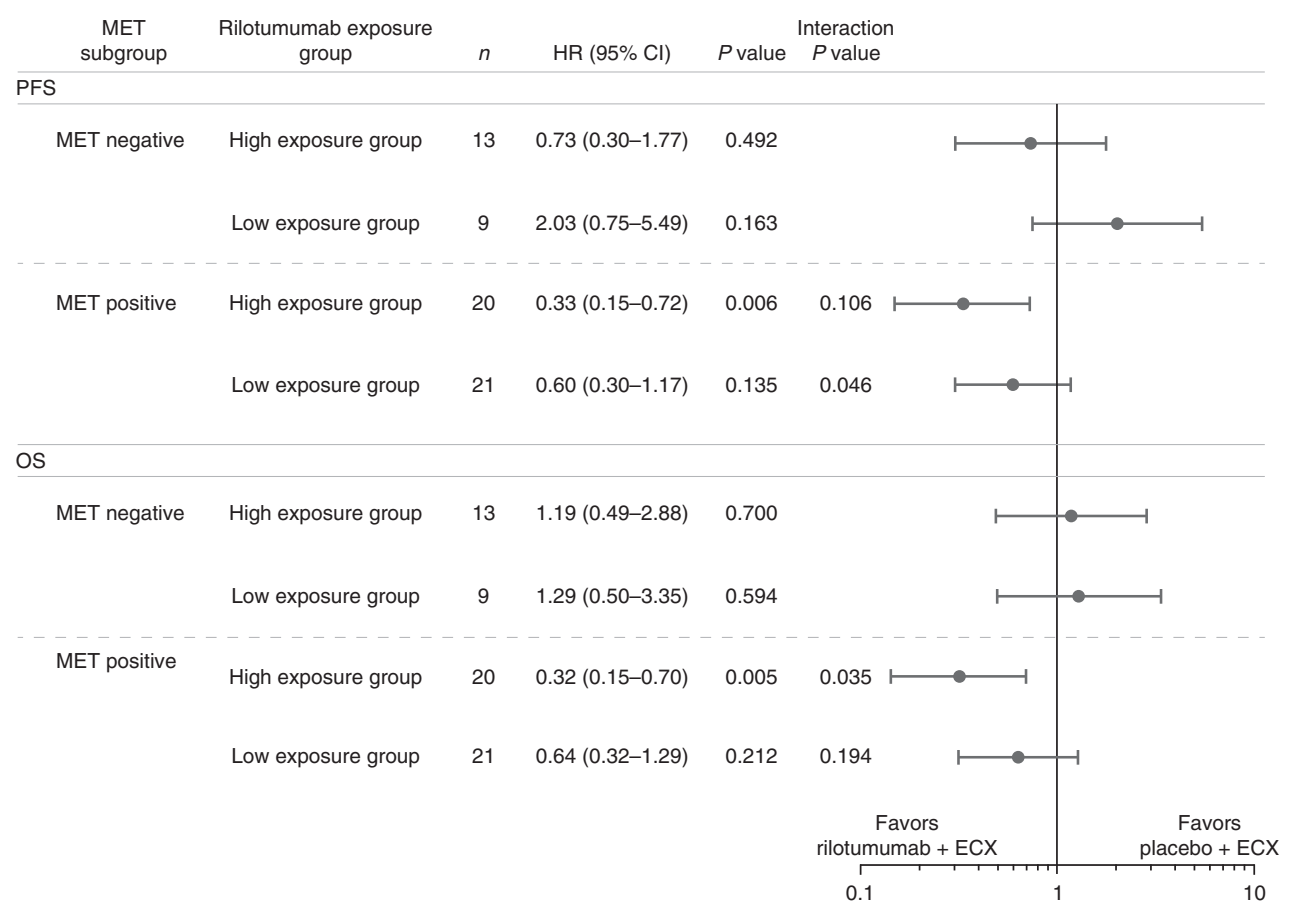

Figure 2. Forest plots for progression-free survival (PFS) and overall survival (OS) with respect to low and high rilotumumab exposure and positive and negative tumour MET expression. Low rilotumumab exposure was defined as $C_{\text {minss }}<94 \mu \mathrm{gl}^{-1}$, and high rilotumumab exposure was defined as $C_{\text {minss }} \geqslant 94 \mu \mathrm{g} \mathrm{ml}^{-1}$. MET positivity was defined as $\geqslant 25 \%$ membranous staining of tumour cells at any intensity, and MET negativity was defined as $<25 \%$ membranous staining. $\mathrm{Cl}$, confidence interval; ECX, epirubicin, cisplatin, and capecitabine; HR, hazard ratio.
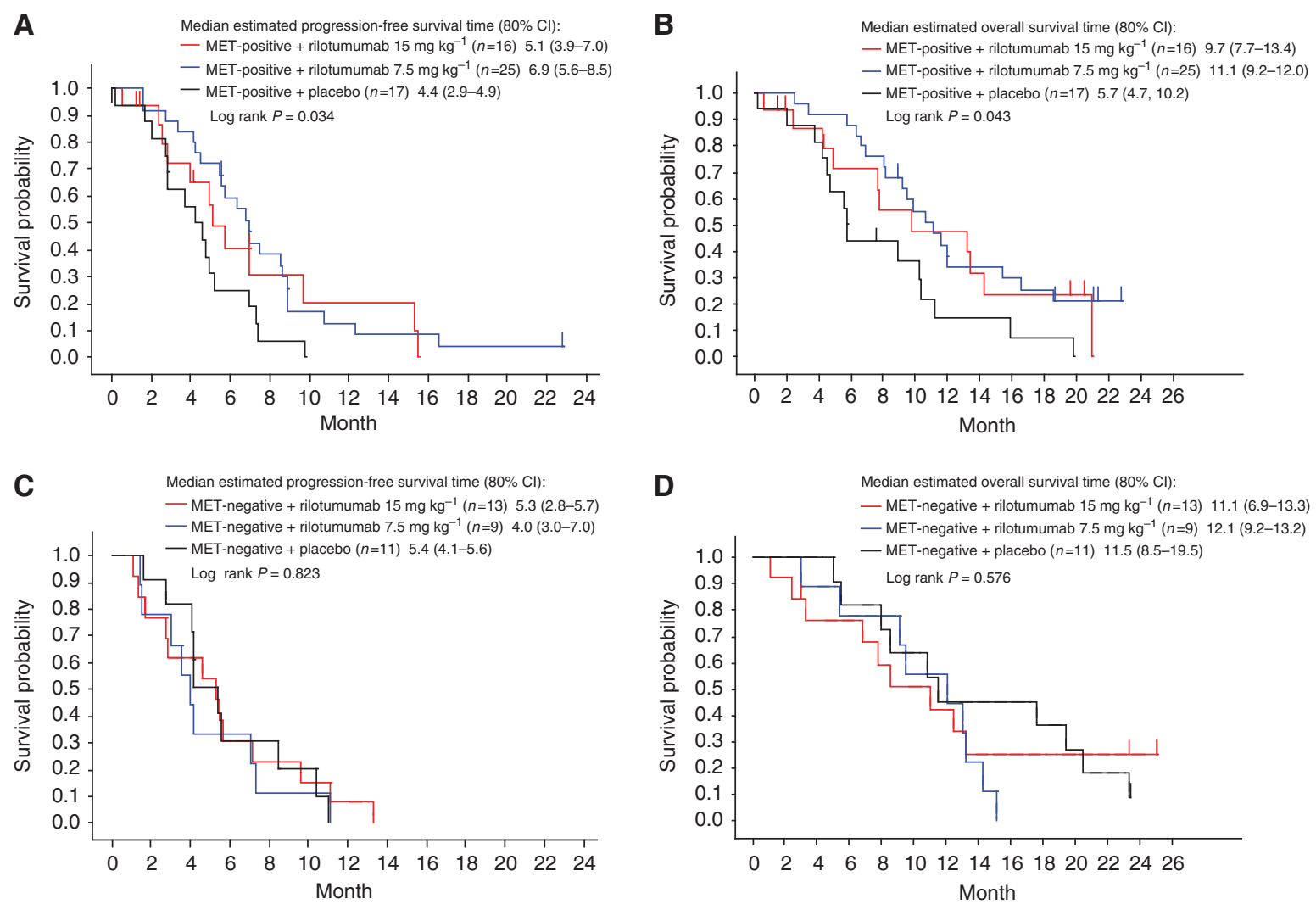

Figure 3. Kaplan-Meier analysis of progression-free survival (PFS) and overall survival (OS). PFS is shown in A and C. OS is shown in B and D. PFS and OS were examined based on rilotumumab dose in the MET-positive (A, B) and MET-negative subgroups (C, D). MET positivity was defined as $\geqslant 25 \%$ membranous staining of tumour cells at any intensity, and MET negativity was defined as $<25 \%$ membranous staining. $\mathrm{Cl}$, confidence interval. 
95\% CI $=0.17-0.60 ; P<0.001)$. Rilotumumab had less of an effect on OS in the low rilotumumab exposure group compared with the placebo group $(\mathrm{HR}=0.47 ; 95 \% \mathrm{CI}=0.25-0.86 ; P=0.014)$. This analysis, in conjunction with the exposure-MET-survival analysis, suggests that rilotumumab has a concentration-dependent treatment effect in patients with MET-positive gastric or oesophagogastric junction cancer.

Exposure-safety analysis. To further explore the exposureresponse relationship, patient incidence of AEs (all grades) was examined among patients in the placebo and low and high rilotumumab exposure groups, and we did not find any apparent patterns of exposure-AE relationships for most AEs. Nevertheless, the incidence of grade $\geqslant 3$ neutropenia trended higher in patients with high rilotumumab exposure $(51.2 \%)$ compared with the placebo group (28\%; Table 3$)$. In an exploratory analysis, no apparent association was observed between rilotumumab exposure and changes in laboratory values of interest from baseline. The combination of rilotumumab and ECX appeared to have a manageable safety profile, regardless of rilotumumab exposure.

\section{DISCUSSION}

Rilotumumab inhibits the MET pathway, which may play a critical role in cell proliferation, survival, and migration in MET-dependent tumours. In this study as well as in previous studies (Taniguchi et al, 1998; Wu et al, 1998; Nakajima et al, 1999; Birchmeier et al, 2003; Drebber et al, 2008; Lennerz et al, 2011; Iveson et al, 2014), tumour MET expression appears to be a prognostic factor for worse survival. In the placebo group, the median OS of patients with MET-positive tumours was approximately $50 \%$ of that of patients with METnegative tumours. Among patients with MET-negative tumours, rilotumumab did not appear to have an effect; the median OS values for the placebo and rilotumumab groups were comparable. This suggests that rilotumumab and ECX may only be effective on gastric or oesophagogastric junction tumours that are highly dependent on the MET pathway for growth. An exposure-survival relationship was seen among patients with MET-positive tumours, indicating that the treatment effect size was associated with plasma rilotumumab concentrations. Both tumour MET levels and rilotumumab exposure must be considered when selecting an effective clinical dose.
Higher rilotumumab exposure does not appear to be associated with an increased incidence of most AEs. Of note, this exposuresafety analysis was not adjusted for time on treatment. If patients in the high-exposure group survived longer, they would be exposed to rilotumumab for a longer period than patients with low exposure or patients in the placebo arm. Thus, the incidence of AEs may be confounded.

Together, the exposure-biomarker-survival analyses support evaluating patients with MET-positive gastric or oesophagogastric junction tumours and dosing to $15 \mathrm{mg} \mathrm{kg}^{-1}$ of rilotumumab in a phase 3 trial, as this dose can provide the required rilotumumab exposure level in most patients. To further verify the findings from this analysis, rilotumumab exposure levels and tumour MET levels will be measured in all patients participating in the phase 3 trial, and exposure-MET-survival analyses have been planned.

No dose-dependent-survival relationship was observed in the phase 2 rilotumumab gastric cancer trial (Iveson et al, 2014). In the subgroup analyses presented here, a survival benefit was observed with rilotumumab among patients with MET-positive tumours, but again, no clear dose-response relationship was observed. Thus, rilotumumab exposure, rather than dose, was most strongly associated with a survival benefit. Multiple factors may contribute to this finding. Primarily, the phase 2 trial did not include tumour MET expression as one of the randomisation factors, and the distribution of patients with MET-negative and MET-positive tumours was unequal among arms (Iveson et al, 2014). Also, the phase 2 study was not powered to compare the efficacy between the two dose arms. Last, high inter-patient variability in rilotumumab exposure was seen, as is common in antibody therapies. The ranges of rilotumumab exposure partially overlapped among patients receiving 7.5 and $15 \mathrm{mg} \mathrm{kg}^{-1}$ rilotumumab, and patients receiving the lower dose may not necessarily have had a lower drug exposure. Thus, comparing exposure is a more sensitive test of the importance of drug levels on outcome because each patient can serve as an individual data point as opposed to being grouped with all other patients who received a given dose, some of whom may have had an exposure more consistent with the other dose group.

Traditionally, dose-ranging studies are used to determine the optimal dose of an investigational drug in early clinical trials to maximise efficacy while maintaining a manageable safety profile (Ratain et al, 2008). As phase 2 oncology trials are often not powered to investigate a broad dose range, a clear dose-dependent

Table 3. Treatment-emergent adverse events

\begin{tabular}{|c|c|c|c|c|}
\hline & Placebo $(\mathbf{N}=39)$ & $\begin{array}{l}\text { Low rilotumumab } \\
\text { exposure }(N=40)\end{array}$ & $\begin{array}{l}\text { High rilotumumab } \\
\text { exposure }(N=41)\end{array}$ & Overall $(N=120)$ \\
\hline Any $\mathrm{AE}$ & $39(100.0)$ & $39(97.5)$ & $41(100.0)$ & 119 (99.2) \\
\hline Grade $\geqslant 3$ & $29(74.4)$ & $36(90.0)$ & $35(85.4)$ & $100(83.3)$ \\
\hline Serious AEs & $20(51.3)$ & $25(62.5)$ & $22(53.7)$ & $67(55.8)$ \\
\hline Fatal AEs & $6(15.4)$ & $5(12.5)$ & $4(9.8)$ & $15(12.5)$ \\
\hline \multicolumn{5}{|l|}{ Common grade $\geqslant 3 \mathrm{AEs}^{\mathrm{a}}$} \\
\hline $\begin{array}{l}\text { Neutropenia } \\
\text { Anaemia } \\
\text { Fatigue } \\
\text { Vomiting } \\
\text { Diarrhoea } \\
\text { Palmar-plantar erythrodysesthesia syndrome } \\
\text { Abdominal pain } \\
\text { Hypokalemia } \\
\text { Dehydration } \\
\text { Pulmonary embolism } \\
\text { Nausea } \\
\text { Febrile neutropenia } \\
\text { Deep vein thrombosis }\end{array}$ & $\begin{aligned} & 11(28.2) \\
& 5(12.8) \\
& 6(15.4) \\
& 4(10.3) \\
& 2(5.1) \\
& 2(5.1) \\
& 3(7.7) \\
& 3(7.7) \\
& 3(7.7) \\
& 4(10.3) \\
& 3(7.7) \\
& 2(5.1) \\
& 0(0.0)\end{aligned}$ & $\begin{aligned} 15 & (37.5) \\
5 & (12.5) \\
7 & (17.5) \\
4 & (10.0) \\
2 & (5.0) \\
4 & (10.0) \\
1 & (2.5) \\
3 & (7.5) \\
2 & (5.0) \\
5 & (12.5) \\
3 & (7.5) \\
4 & (10.0) \\
5 & (12.5)\end{aligned}$ & $\begin{aligned} & 21(51.2) \\
& 7(17.1) \\
& 3(7.3) \\
& 3(7.3) \\
& 2(4.9) \\
& 3(7.3) \\
& 3(7.3) \\
& 3(7.3) \\
& 2(4.9) \\
& 2(4.9) \\
& 2(4.9) \\
& 2(4.9) \\
& 2(4.9)\end{aligned}$ & $\begin{array}{c}47(39.2) \\
17(14.2) \\
16(13.3) \\
11(9.2) \\
6(5.0) \\
9(7.5) \\
7(5.8) \\
9(7.5) \\
7(5.8) \\
11(9.2) \\
8(6.7) \\
8(6.7) \\
7(5.8) \\
\end{array}$ \\
\hline
\end{tabular}


drug effect on patient outcomes may not be observed. Exposureresponse analysis may partially overcome this limitation as it accounts for the drug disposition and the drug exposure level in each individual patient, although the patients might receive the same dose (Workman, 2002; Wetherington et al, 2010). However, exposure-response analyses may not be better than the doseresponse analyses with respect to the selection of patients for the targeted treatment, as the level of the target may vary among patients. Therefore, inclusion of relevant pathway biomarkers could strengthen the exposure-response analysis.

Initial dose-survival analysis (Figure 1A and B) did not take into account individual differences in drug exposure or tumour MET expression, and no clear dose-dependent-response relationship was observed. Although the relationship between rilotumumab exposure and patient outcomes was clearer in the exposureresponse analysis (Figure $1 \mathrm{C}$ and D), individual differences in MET expression were still not considered. Finally, the exposure-survival analyses in the MET-positive and MET-negative subgroups (Figure $1 \mathrm{E}$ and $\mathrm{H}$ ) demonstrated the effect of MET expression and drug exposure on survival.

Identifying relevant biomarkers and including these biomarkers in exposure-response analyses should be applied to future exposureresponse analyses whenever possible. The identification of predictive biomarkers and relevant pharmacodynamic markers for survival is not straightforward. A lack of understanding of the biology of the target and its relationship to the disease contributes to this challenge. Furthermore, well-characterised tests for quantifying potential biomarkers are needed so that results can be better analysed, and findings should be confirmed in larger clinical trials.

This study had several limitations. First, the exposurebiomarker-survival analysis had small sample sizes in the subgroups, thus limiting the interpretation of the results. Second, although the analysis plan was pre-specified before the primary analysis was conducted, the analysis is considered retrospective and exploratory. Although these limitations may increase the chances of falsely obtaining significant subgroup effects and interactions (Dijkman et al, 2009), the results of the subgroup analysis were consistent with the overall analysis and current knowledge of the MET pathway (Taniguchi et al, 1998; Nakajima et al, 1999; Cao et al, 2001; Drebber et al, 2008; Lennerz et al, 2011).

In conclusion, we observed that patients with high rilotumumab exposure and MET-positive tumours had longer survival than those with low rilotumumab exposure or MET-negative tumours. They appeared to benefit the most from rilotumumab plus ECX treatment. The safety results were generally similar between the low- and high-exposure subgroups, with the exception of grade $\geqslant 3$ neutropenia that was more frequent with high exposure. Considering the small sample size and retrospective nature of our analyses, our findings need to be confirmed in future trials.

\section{ACKNOWLEDGEMENTS}

We thank the patients, investigators, and the medical, nursing, and laboratory staff who participated in the rilotumumab clinical trials; Mark Ma and Teresa Wong of Amgen Inc. for support of the analytical assay development and sample analysis; and Kerri Hebard-Massey and Jenilyn Virrey of Amgen Inc. for medical writing assistance. This study was funded by Amgen Inc.

\section{CONFLICT OF INTEREST}

MZ, RT, S Doshi, KSO, YZ, S Dubey, YJ, EYL, and TI are employees of and stockholders in Amgen Inc. RCD has no conflict of interest to disclose.

\section{REFERENCES}

Accornero P, Pavone LM, Baratta M (2010) The scatter factor signaling pathways as therapeutic associated target in cancer treatment. Curr Med Chem 17: 2699-2712.

Amemiya H, Kono K, Itakura J, Tang RF, Takahashi A, An FQ, Kamei S, Iizuka H, Fujii H, Matsumoto Y (2002) c-Met expression in gastric cancer with liver metastasis. Oncology 63: 286-296.

Beppu K, Uchiyama A, Morisaki T, Nakamura K, Noshiro H, Matsumoto K, Nakamura T, Tanaka M, Katano M (2000) Elevation of serum hepatocyte growth factor concentration in patients with gastric cancer is mediated by production from tumor tissue. Anticancer Res 20: 1263-1267.

Birchmeier C, Birchmeier W, Gherardi E, Vande Woude GF (2003) Met, metastasis, motility and more. Nat Rev Mol Cell Biol 4: 915-925.

Bruno R, Lu JF, Sun YN, Claret L (2011) A modeling and simulation framework to support early clinical drug development decisions in oncology. J Clin Pharmacol 51: 6-8.

Burgess T, Coxon A, Meyer S, Sun J, Rex K, Tsuruda T, Chen Q, Ho SY, Li L, Kaufman S, McDorman K, Cattley RC, Sun J, Elliott G, Zhang K, Feng X, Jia XC, Green L, Radinsky R, Kendall R (2006) Fully human monoclonal antibodies to hepatocyte growth factor with therapeutic potential against hepatocyte growth factor/c-Met-dependent human tumors. Cancer Res 66: $1721-1729$.

Cao B, Su Y, Oskarsson M, Zhao P, Kort EJ, Fisher RJ, Wang LM, Vande Woude GF (2001) Neutralizing monoclonal antibodies to hepatocyte growth factor/scatter factor (HGF/SF) display antitumor activity in animal models. Proc Natl Acad Sci USA 98: 7443-7448.

Claret L, Lu JF, Bruno R, Hsu CP, Hei YJ, Sun YN (2012) Simulations using a drug-disease modeling framework and phase II data predict phase III survival outcome in first-line non-small-cell lung cancer. Clin Pharmacol Ther 92: 631-634.

Claret L, Lu JF, Sun YN, Bruno R (2010) Development of a modeling framework to simulate efficacy endpoints for motesanib in patients with thyroid cancer. Cancer Chemother Pharmacol 66: 1141-1149.

Dijkman B, Kooistra B, Bhandari M. Evidence-Based Surgery Working G (2009) How to work with a subgroup analysis. Can J Surg 52: 515-522.

Drebber U, Baldus SE, Nolden B, Grass G, Bollschweiler E, Dienes HP, Hölscher AH, Mönig SP (2008) The overexpression of c-met as a prognostic indicator for gastric carcinoma compared to p53 and p21 nuclear accumulation. Oncol Rep 19: 1477-1483.

Gao CF, Xie Q, Zhang YW, Su Y, Zhao P, Cao B, Furge K, Sun J, Rex K, Osgood T, Coxon A, Burgess TL, Vande Woude GF (2009) Therapeutic potential of hepatocyte growth factor/scatter factor neutralizing antibodies: inhibition of tumor growth in both autocrine and paracrine hepatocyte growth factor/scatter factor:c-Met-driven models of leiomyosarcoma. Mol Cancer Ther 8: 2803-2810.

Gordon MS, Sweeney CS, Mendelson DS, Eckhardt SG, Anderson A, Beaupre DM, Branstetter D, Burgess TL, Coxon A, Deng H, Kaplan-Lefko P, Leitch IM, Oliner KS, Yan L, Zhu M, Gore L (2010) Safety, pharmacokinetics, and pharmacodynamics of AMG 102, a fully human hepatocyte growth factor-neutralizing monoclonal antibody, in a first-in-human study of patients with advanced solid tumors. Clin Cancer Res 16: 699-710.

Han SU, Lee JH, Kim WH, Cho YK, Kim MW (1999) Significant correlation between serum level of hepatocyte growth factor and progression of gastric carcinoma. World J Surg 23: 1176-1180.

Iveson T, Donehower RC, Davidenko I, Tjulandin S, Deptala A, Harrison M, Nirni S, Lakshmaiah K, Thomas A, Jiang Y, Zhu M, Tang R, Anderson A, Dubey S, Oliner KS, Loh E (2014) Rilotumumab in combination with epirubicin, cisplatin, and capecitabine as first-line treatment for gastric or oesophagogastric junction adenocarcinoma: an open-label, dose de-escalation phase $1 \mathrm{~b}$ study and a double-blind, randomised phase 2 study. Lancet Oncol 15: 1007-1018.

Janjigian YY, Tang LH, Coit DG, Kelsen DP, Francone TD, Weiser MR, Jhanwar SC, Shah MA (2011) MET expression and amplification in patients with localized gastric cancer. Cancer Epidemiol Biomarkers Prev 20: 1021-1027.

Jun HT, Sun J, Rex K, Radinsky R, Kendall R, Coxon A, Burgess TL (2007) AMG 102, a fully human anti-hepatocyte growth factor/scatter factor neutralizing antibody, enhances the efficacy of temozolomide or docetaxel in U-87 MG cells and xenografts. Clin Cancer Res 13: 6735-6742.

Lennerz JK, Kwak EL, Ackerman A, Michael M, Fox SB, Bergethon K, Lauwers GY, Christensen JG, Wilner KD, Haber DA, Salgia R, Bang YJ, 
Clark JW, Solomon BJ, Iafrate AJ (2011) MET amplification identifies a small and aggressive subgroup of esophagogastric adenocarcinoma with evidence of responsiveness to crizotinib. J Clin Oncol 29: 4803-4810.

Maulik G, Kijima T, Ma PC, Ghosh SK, Lin J, Shapiro GI, Schaefer E, Tibaldi E, Johnson BE, Salgia R (2002) Modulation of the c-Met/ hepatocyte growth factor pathway in small cell lung cancer. Clin Cancer Res 8: 620-627.

Nakajima M, Sawada H, Yamada Y, Watanabe A, Tatsumi M, Yamashita J, Matsuda M, Sakaguchi T, Hirao T, Nakano H (1999) The prognostic significance of amplification and overexpression of c-met and c-erb B-2 in human gastric carcinomas. Cancer 85: 1894-1902.

Nishiyama T, Sasaki T, Takaishi K, Kato M, Yaku H, Araki K, Matsuura Y, Takai Y (1994) rac p21 is involved in insulin-induced membrane ruffling and rho p21 is involved in hepatocyte growth factor- and 12-Otetradecanoylphorbol-13-acetate (TPA)-induced membrane ruffling in KB cells. Mol Cell Biol 14: 2447-2456.

Ratain MJ, Humphrey RW, Gordon GB, Fyfe G, Adamson PC, Fleming TR, Stadler WM, Berry DA, Peck CC (2008) Recommended changes to oncology clinical trial design: revolution or evolution? Eur J Cancer 44: $8-11$.

Tanaka K, Miki C, Wakuda R, Kobayashi M, Tonouchi H, Kusunoki M (2004) Circulating level of hepatocyte growth factor as a useful tumor marker in patients with early-stage gastric carcinoma. Scand J Gastroenterol 39: 754-760.

Taniguchi K, Yonemura Y, Nojima N, Hirono Y, Fushida S, Fujimura T, Miwa K, Endo Y, Yamamoto H, Watanabe H (1998) The relation between the growth patterns of gastric carcinoma and the expression of hepatocyte growth factor receptor (c-met), autocrine motility factor receptor, and urokinase-type plasminogen activator receptor. Cancer 82: 2112-2122.

Taniguchi T, Kitamura M, Arai K, Iwasaki Y, Yamamoto Y, Igari A, Toi M (1997) Increase in the circulating level of hepatocyte growth factor in gastric cancer patients. Br J Cancer 75: 673-677.

Wetherington JD, Pfister M, Banfield C, Stone JA, Krishna R, Allerheiligen S, Grasela DM (2010) Model-based drug development: strengths, weaknesses, opportunities, and threats for broad application of pharmacometrics in drug development. J Clin Pharmacol 50: 31S-46S.

Workman P (2002) Challenges of PK/PD measurements in modern drug development. Eur J Cancer 38: 2189-2193.

Wu CW, Li AF, Chi CW, Chung WW, Liu TY, Lui WY, P’Eng FK (1998) Hepatocyte growth factor and Met/HGF receptors in patients with gastric adenocarcinoma. Oncol Rep 5: 817-822.

Zhu M, Doshi S, Gisleskog PO, Oliner KS, Perez Ruixo JJ, Loh E, Zhang Y (2014) Population pharmacokinetics of rilotumumab, a fully human monoclonal antibody against hepatocyte growth factor, in cancer patients. J Pharm Sci 103: 328-336.

This work is published under the standard license to publish agreement. After 12 months the work will become freely available and the license terms will switch to a Creative Commons AttributionNonCommercial-Share Alike 4.0 Unported License. 